\title{
Prophylactic Use of Antiepileptic Drug (Phenytoin) in Preventing Early Postoperative Seizure in Patients with Chronic Subdural Hematoma: A Randomized Control Trial
}

\author{
Amit Bahadur Pradhanang ${ }^{1}$ Gopal Sedain ${ }^{1, \oplus}$ Sushil K. Shilpakar ${ }^{1} \quad$ Mohan Raj Sharma ${ }^{1}$ \\ ${ }^{1}$ Department of Neurosurgery, Institute of Medicine, Tribhuvan \\ University Teaching Hospital, Kathmandu, Nepal \\ Address for correspondence Gopal Sedain, MS, MCh, Department \\ of Neurosurgery, Institute of Medicine, Tribhuvan University Teaching \\ Hospital, Maharajgunj, Kathmandu, $44600 \mathrm{Nepal}$ \\ (e-mail: newron79@gmail.com).
}

Indian J Neurosurg 2019;8:168-178

\begin{abstract}
Keywords

- chronic SDH

- phenytoin

- seizure

Introduction Antiepileptic drug (AED) therapy has been used prophylactically in various centers in patients with chronic subdural hematoma (CSDH) after surgical evacuation, although the benefit is unclear.

Materials and Methods In this randomized controlled trial, patients with CSDH undergoing burr hole drainage were enrolled into two groups-one which received prophylactic phenytoin and the other without the drug use. Their clinical presentation, imaging findings and postoperative complications were analyzed. Primary objective of the study was to assess the efficacy of phenytoin in the reduction of early postoperative seizure.

Results A total of 54 patients were enrolled in the study and randomized into two groups. Twenty-seven patients were assigned into the phenytoin group while another 27 patients to the "no phenytoin" group. Analysis of all patients in the no phenytoin group and those in the phenytoin group with therapeutic serum phenytoin levels were done. The incidence of postoperative seizure was 5.7\%; and all patients who had seizures were in the no phenytoin group. This, however, was not statistically significant $(p=0.262)$. Two out of 25 patients on phenytoin ( $8 \%)$ developed adverse drug reactions. Conclusion Incidence of postoperative seizure in patients undergoing burr hole drainage for CSDH was low. Routine prophylactic use of AED, did not reduce seizure in early postoperative period.
\end{abstract}

\section{Introduction}

Chronic subdural hematoma (CSDH) is a common neurosurgical entity. ${ }^{1}$ It primarily affects the elderly age group and is a cause of considerable morbidity and mortality. ${ }^{2,3}$ The reported incidence ranges from 3.4 per 100,000 in populations younger than 65 years to $8-58$ per 100,000 in those older than 65 years. ${ }^{4-6}$

Chronic subdural hematomas are usually caused by minor head injuries; and majority are believed to result from tearing of bridging veins. ${ }^{7}$ Burr hole drainage by one or two craniostomies is the surgical procedure performed most commonly for this condition. ${ }^{8,9}$

The frequency of seizures in these patients is not fully established. The incidence of postoperative seizures is reported to be between $1 \%$ and $32 \% .{ }^{10-12}$

The efficacy of prophylactic antiepileptic drug (AED) in this pathology has been debated. Ohno et al, in 1993, ${ }^{13}$ reported that the low incidence of seizures does not justify an anticonvulsant prophylaxis in patients with CSDH caused received

May 20, 2019

accepted

July 29, 2019

published online

September 24, 2019
DOI https://doi.org/

10.1055/s-0039-1698478

ISSN 2277-954X.
C2019 Neurological Surgeons'

Society of India
License terms

(c) $1 \$$ 
by minor head injury. Similarly, a study by Rubin et al, in $1993,{ }^{14}$ suggested a low incidence of seizure and that this prophylactic medication should be considered only in alcoholic patients because of their higher risk of seizure. Whereas a study by Sabo et al, in $1995,{ }^{12}$ noted a high incidence of postoperative seizures in patients treated for CSDH and a significant increase in morbidity and mortality associated with respiratory complications and status epilepticus in patients with new-onset seizures; they recommended the administration of AED for a period of 6 months following diagnosis in all patients with CSDH. However, all of these studies were retrospective in nature.

A Cochrane review by Ratilal et al, in $2013,{ }^{15}$ concluded that no formal recommendation could be made on the use of prophylactic anticonvulsants in patients with CSDH. There are no randomized controlled trials on the topic till date.

\section{General Objectives}

- To assess the efficacy of phenytoin in preventing early postoperative seizure.

\section{Specific Objectives}

1. To define the demographic characteristics and clinical manifestations of patients with CSDH.

2. To assess the adverse side effects of phenytoin.

3. To assess the functional outcome of patients following burr hole drainage using Glasgow Outcome Scale (GOSE) and modified Rankin Scale (MRS).

4. To assess other complications: permanent neurological impairment and mortality in patients with CSDH.

\section{Outcome Measures}

\section{Primary Outcome}

- Seizure occurrence in the early postoperative period

\section{Secondary Outcomes}

- Demographic characteristics of patients with CSDH

- Specific AED adverse drug reaction

- Immediate postoperative complications

- Mortality

- Long-term complications: permanent neurological impairment

- Functional status at 6 months MRS

- Functional status at 6 months using Extended GOSE

\section{Prophylactic Anticonvulsants Following CSDH}

Regarding the use of prophylactic administration of AEDs in patients with CSDH, there remains a lack of consensus. The reported rate of seizures in this patient population is substantial but varies widely from 2 to $19 \%{ }^{13,16}$ The administration of AEDs is reasonable, since patients with CSDH frequently exhibit some extent of underlying traumatic brain injury, a well-established risk factor for seizures. ${ }^{17}$ However, anticonvulsant medications have been shown to increase the risk of falling in patients aged 65 years and older, which in itself represents a primary risk factor for SDH. ${ }^{18}$ Therefore, the added risk of recurrent hemorrhage following anticonvulsant treatment along with its well-known adverse effects, must be outweighed by the benefits of AED prophylaxis. Unfortunately, the efficacy of seizure prophylaxis in the setting of both treated and untreated CSDH has been addressed only through a small number of retrospective studies. ${ }^{12-14,19}$

Two studies reported no significant difference in seizure frequency related to the prophylactic administration of AEDs. ${ }^{13,14}$ Both groups concluded that the morbidity of AED administration outweighed the benefits except in patients at particularly high risk of developing seizures, such as chronic consumers of alcohol. ${ }^{13,14}$ In contrast, Sabo et al demonstrated that while $32 \%$ of the patients receiving inadequate prophylaxis developed seizures, only $2 \%$ patients receiving appropriate prophylaxis developed new seizures..$^{12}$ This study also demonstrated a significant correlation between seizure activity and both morbidity and mortality. Although these three studies reached seemingly contradictory conclusions, their experimental design varied significantly; concrete conclusions are difficult to draw from their results. A study published by Bartosz et al, ${ }^{19}$ on the other hand, examined the effect of timing of AED prophylaxis. They found that preoperative initiation of AEDs was the only independent predictor of decreased postoperative seizure incidence. However, the study found no effect of the timing of AED initiation on discharge outcomes.

\section{Study Design}

The study design was a randomized controlled trial (RCT).

\section{Study Setting}

This study was conducted at the Neurosurgical Unit of Department of Surgery, Tribhuvan University Teaching Hospital (TUTH), Kathmandu, Nepal. TUTH is a tertiary care center located in Kathmandu, with a well-established neurosurgical department.

\section{Study Population}

All patients diagnosed with CSDH and requiring surgical evacuation with burr hole drainage were enrolled in the study. It was completed over a period of 1 year, starting from November 2013 to November 2014.

\section{Sample Size}

The anticipated incidence in the phenytoin group was $2.4 \%$ (P2), while it was $32 \%$ in the no phenytoin group (P1). Using Z $\alpha$ as 1.96 at $95 \%$ confidence interval and $Z \beta$ as 0.84 at $80 \%$ power, the sample size was calculated as per the following formula: 
$\mathrm{P} 1=0.32$ in the study group

$\mathrm{P} 2=0.024$ in the other group

$$
\mathrm{n}=\left[\frac{Z_{a} \sqrt{2 p(1-p)}+Z_{\beta} \sqrt{\mathrm{p}_{1}\left(1-p_{1}\right)+p_{2}\left(1-p_{2}\right)}}{\left(p_{1}-p_{2}\right)}\right]^{2}
$$

$\mathrm{P}=(\mathrm{P} 1+\mathrm{P} 2) / 2$

The sample size, including a $10 \%$ dropout rate, was calculated to be 27 in each group.

\section{Data Collection Tools and Techniques Supervision and Monitoring}

- During intravenous use of Phenytoin

- ECG and blood pressure were monitored continuously.

- During hospital stay

- Family members and nursing staff were trained to identify occurrence of seizure activity and instructed to promptly inform the neurosurgeon on call. These patients received AED as per the study protocol.

\section{- At discharge}

- Care providers were requested to document and promptly bring the patient to the nearest health care facility in case of seizure occurrence and also inform the investigator via telephone.

- Family members of the patients receiving phenytoin were also trained to recognize the specific adverse effects of the drug and instructed to stop it immediately and report to nearby health facility and the investigator if such adverse effects developed.

- Patients were asked to follow-up on day 7 of the surgery and serum levels of those patients on phenytoin were determined. Later, a follow-up was scheduled at 6 months postsurgery. In these visits, documentation of seizure and performance scores was done.

\section{Randomization}

Computer-generated randomization table was used for this purpose.

\section{Treatment Protocol}

Patients were assigned to either of the two groups (phenytoin or no phenytoin) on arrival in the emergency department. Patients with drug allergy to phenytoin were excluded from the study. Patients in the phenytoin group were administered a loading dose of intravenous phenytoin at a dose of $17 \mathrm{mg} / \mathrm{kg}$. The total loading dose of phenytoin was calculated and added in $100 \mathrm{~mL}$ of $0.9 \%$ saline and given as an intravenous infusion over 30 to 60 minutes under continuous ECG and blood pressure monitoring. It was continued at a dose of $100 \mathrm{mg}$ administered three times a day or $300 \mathrm{mg}$ daily for a total of 7 days after surgery. Patients in the no phenytoin group did not receive any AEDs.

Burr hole drainage was performed in a standard fashion by the neurosurgical team in the operation theater under local anesthesia in majority of the cases and only few cases were performed under general anesthesia as the condition of the patient necessitated.

\section{Data Processing and Analysis}

Data analysis was done as per the objectives of the study. Coding and entry of collected data were done in Epi data 3.1. Analysis of data was performed in version 20 of Statistical Package for Social Sciences (SPSS) software. Descriptive analyses were conducted to present general information of the study population. Data analysis was done by frequency run and cross-tabulations. Categorical variables were presented as percentage and frequency, whereas continuous variables were presented as mean and median. Bivariate and multivariate analyses were done to find the association between outcome and explorative variables. In bivariate analysis, Chi-square test (or Fisher exact test) was applied to test the significance of association between independent and dependent variables. Odds ratio with $95 \%$ confidence interval $(\mathrm{CI})$ was used to test the strength of the association.

\section{Inclusion Criteria}

All patients diagnosed as CSDH, based on CT/MRI of head who were candidates for surgical intervention.

\section{Exclusion Criteria}

- Patients allergic to phenytoin

- History of pre-existing seizures

- Age < 14 years

- Associated intraparenchymal lesion seen on CT scan of head at presentation

- Patients already on AED

- Patients refusing to participate in the study

\section{Ethical Consideration}

Ethical clearance was obtained from the institutional ethical review board (IRB) of the Institute of Medicine, Tribhuvan University, Kathmandu, Nepal.

\section{Results}

A total of 54 patients were randomized into two groups (27 in each group). Twenty-five patients in the phenytoin group with therapeutic serum level of phenytoin and 27 patients in the no phenytoin group were analyzed. Two patients in the phenytoin group with subtherapeutic serum levels of the drug were excluded from the study. As depicted in - Fig. 1, a total of 52 cases with CSDH were analyzed.

Forty-five patients (86.5\%) were males and 7 (13.4\%) females with a male: female ratio of 6.4:1, suggesting an obvious male predilection. The age ranged from 27 to 87 years with the mean age of 64.25 years \pm 14.04 . The 71 to 80 years age group was the largest ( $\boldsymbol{- \text { Fig. }}$ 2). Baseline characteristics in both the groups were comparable, showing no significant differences, as shown in - Table 1.

Since most of our patients were elderly, a large proportion of them had other comorbidities. Hypertension was found in a large proportion of patients (26.9\%). Chronic obstructive 


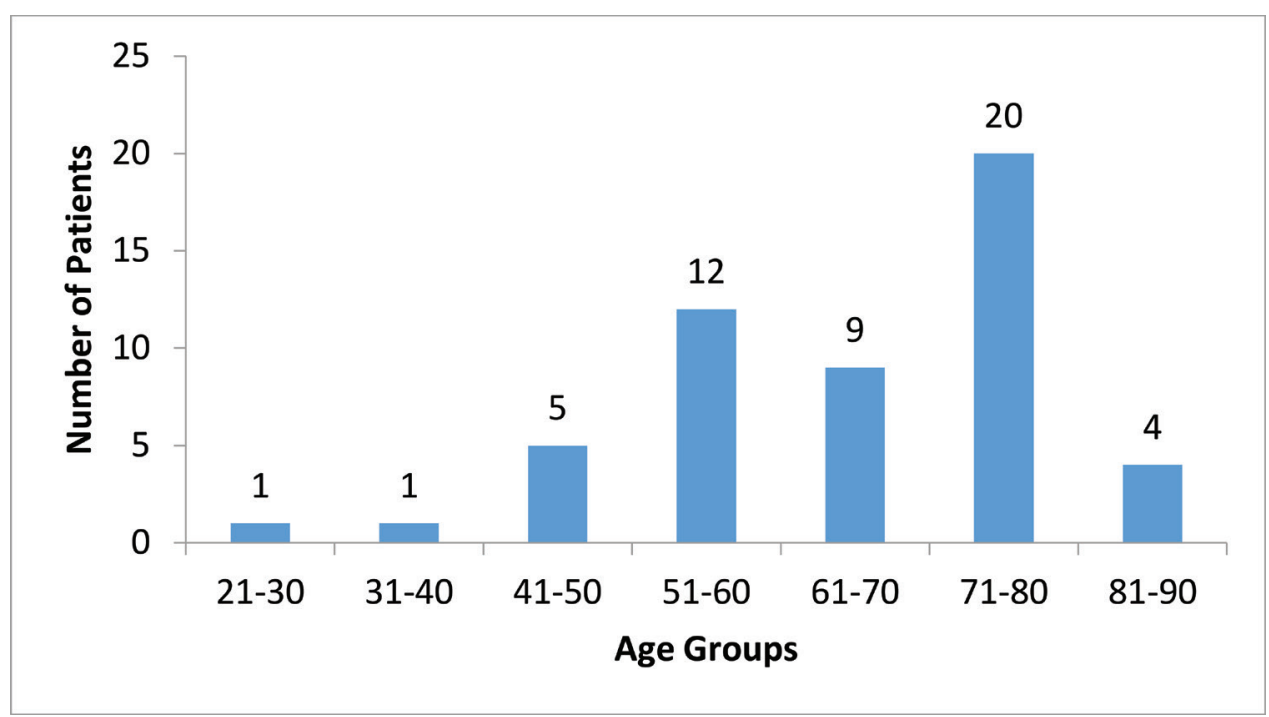

Fig. 1 Study flowchart.

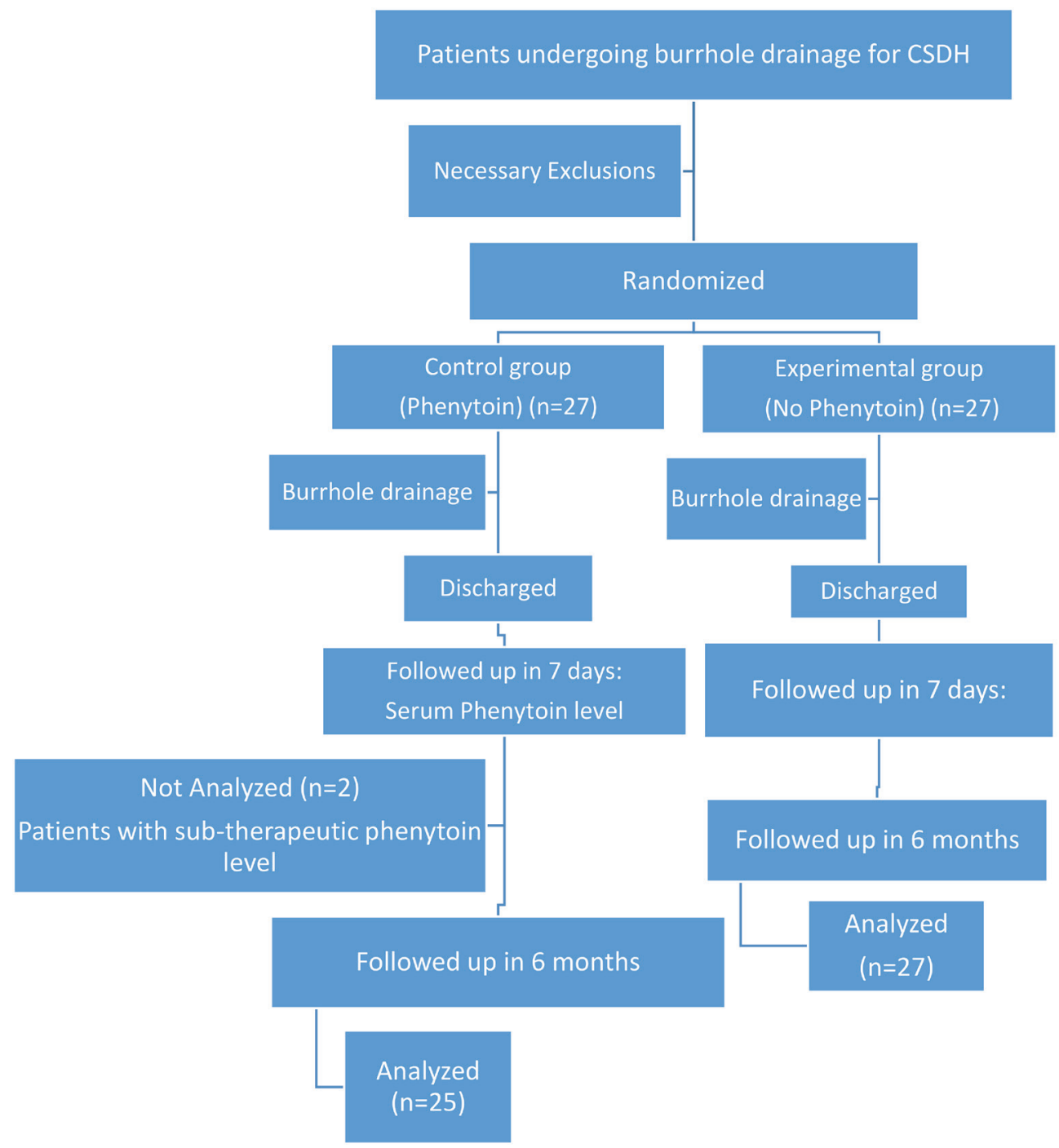

Fig. 2 Age distribution $(n=52)$. 
Table 1 Comparison of baseline characteristics in both groups $(n=52)$

\begin{tabular}{|c|c|c|c|}
\hline Characteristics & Phenytoin $(n=25)$ & No phenytoin $(n=27)$ & $p$-Value \\
\hline Mean age in $y r \pm S D$ & $66.2 \pm 13.5$ & $62.4 \pm 14.6$ & \\
\hline \multicolumn{3}{|l|}{ Sex } & \\
\hline Male & 20 & 25 & \\
\hline Female & 5 & 2 & \\
\hline \multicolumn{4}{|l|}{ Admission GCS scores } \\
\hline Median & 13 & 13 & \\
\hline \multicolumn{4}{|l|}{ Comorbidities } \\
\hline Hypertension & 8 & 6 & 0.42 \\
\hline Diabetes & 3 & 2 & 0.92 \\
\hline Ischemic heart Disease & 2 & 1 & 0.94 \\
\hline Alcohol use & 9 & 11 & 0.72 \\
\hline Tobacco use & 13 & 11 & 0.41 \\
\hline $\begin{array}{l}\text { Chronic obstructive Airway } \\
\text { disease }\end{array}$ & 8 & 7 & 0.62 \\
\hline \multicolumn{4}{|c|}{ History and clinical manifestations } \\
\hline Trauma & 11 & 14 & 0.57 \\
\hline Headache & 17 & 22 & 0.26 \\
\hline Nausea/vomiting & 3 & 3 & 0.73 \\
\hline Altered sensorium & 12 & 15 & 0.58 \\
\hline Incontinence & 4 & 5 & 0.89 \\
\hline Use of anticoagulants & 2 & 1 & 0.94 \\
\hline Dysphasia & 2 & 3 & 0.92 \\
\hline Motor deficit & 16 & 21 & 0.27 \\
\hline \multicolumn{4}{|l|}{ Laterality of hematoma } \\
\hline Right side & 13 & 12 & 0.58 \\
\hline Left side & 9 & 12 & 0.53 \\
\hline Bilateral & 3 & 3 & 0.73 \\
\hline \multicolumn{4}{|l|}{ Chronicity of hematoma } \\
\hline Chronic & 17 & 16 & 0.51 \\
\hline Subacute & 8 & 11 & 0.51 \\
\hline
\end{tabular}

airway disease (COAD), ischemic heart disease, and diabetes mellitus (DM) were among the other concomitant conditions. Little more than one-third of the patients (38.4\%) gave history of regular alcohol consumption. Three patients (5.7\%) were found to have been using antiplatelet agents, namely aspirin or clopidogrel for cardiac-related diseases.

Less than half (48\%) of the patients could recall a history of trauma to the head. The most common mechanism of head injury was fall.

Regarding preoperative MRS, 26 patients (48.0\%) had a grade of 4 , which meant they had moderately severe disability. Moderate severe disability is described as patient being unable to walk without assistance and unable to attend to own bodily needs without assistance. The next commonest grade was grade 3 , followed by grade 5 ( - Fig. 3 ).

Patients who had a Glasgow Comma Scale (GCS) $<13$ had midline shift $>1 \mathrm{~cm}$ and had some form of focal neurological deficit. These were the only patients who had cranial nerve involvement, namely facial or oculomotor palsies, which were seen in 2 patients (3.8\%).

Postoperative GCS improved drastically in the majority of patients. Forty-eight patients (92.3\%) had a GCS of 13 to 15. Only 4 patients did not improve in the early period. In the phenytoin group, after surgical evacuation, 23 patients had GCS 13 to 15,2 patients had GCS 9 to 12 , and GCS $\leq 8$ was not seen in any patient. In the no phenytoin group, 25 patients had GCS of 13 to 15 , no patient had GCS of 9 to 12 , and two patients had GCS of $\leq 8$. Statistical analysis revealed no significant difference between the groups.

The most common postoperative symptom was headache, which was present in $61.5 \%$ patients, followed by nausea and vomiting seen $7.6 \%$ cases. Comparing both groups, 14 patients had headache in the phenytoin group and 17 cases in the no phenytoin group with a p value of 0.60 . Similarly, 
nausea and vomiting were seen in three patients in phenytoin group and one in no phenytoin group with a $\mathrm{p}$ value of 0.54 (-Table 2).

The most commonly encountered complication following CSDH evacuation in our study was pneumonia which was found in four patients (7.6\%). Three patients from the phenytoin group and one from no phenytoin group developed pneumonia. Other complications included subdural tension pneumocephalus for which one patient required reoperation. One patient developed subdural empyema and underwent craniotomy and evacuation of the subdural empyema. Postoperative seizure following CSDH evacuation was also noted in this particular patient.

Two patients among those who had history of chronic alcohol consumption developed alcohol withdrawal syndrome in the postoperative period. Both of them were from the no phenytoin group.

The overall postoperative seizure incidence was 3 (5.7\%). All three patients were from the no phenytoin group. Statistical analysis did not show any significant difference (-Table 3).
On specific evaluation of characteristics of those patients who had postoperative seizures, it revealed that these patients denied any previous history of head trauma. Two patients had history of regular alcohol consumption; however, it was not statistically significant $(p=0.27)$. All three patients presented with hemiparesis although no change was noted in their consciousness. All had hematoma volumes larger than $100 \mathrm{~mL}$. Among the three, one patient had recurrent generalized tonic clonic seizures in the postoperative period. This patient developed subdural empyema, for whom craniotomy and drainage was performed. The patient died later in ICU on postoperative day 12 . The other two patients had a single episode of focal seizure involving the contralateral side of the CSDH within the first week after surgery, and were started on antiepileptic drugs. On postoperative CT scan, one of the patients had a parenchymal contusion at the operated site. On follow-up, none of the surviving two patients had seizures within 6 months of follow-up period ( - Fig. 4)

Serum phenytoin levels of the patients in the phenytoin group was measured using high-performance liquid chromatography on follow-up visit in outpatient department on day

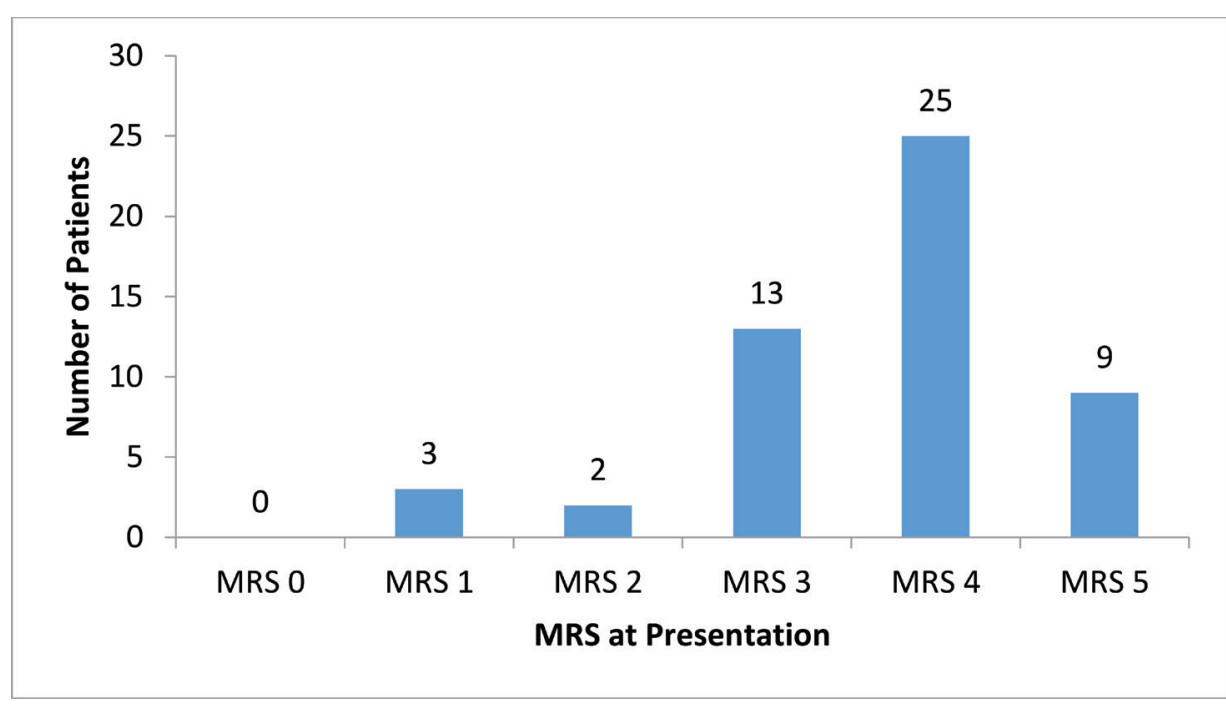

Fig. 3 Modified Rankin Scale at presentation $(n=52)$.

Table 2 Comparison of early postoperative symptoms in patients with CSDH $(n=52)$

\begin{tabular}{|l|l|l|l|}
\hline Postoperative symptom & Phenytoin & No phenytoin & $p$-Value \\
\hline Headache & 14 & 17 & 0.60 \\
\hline Nausea/vomiting & 3 & 1 & 0.54 \\
\hline
\end{tabular}

Table 3 Cross-tabulation comparing rate of occurrence of postoperative seizures in phenytoin and no phenytoin groups following burr hole evacuation of $\mathrm{CSDH}(n=52)$

\begin{tabular}{|l|l|l|l|l|}
\hline Postoperative seizures & Seizure & No seizure & Total & -Value \\
\hline Group & 0 & 25 & 25 & 0.262 \\
\hline Phenytoin & 3 & 24 & 27 & \\
\hline No phenytoin & 3 & 49 & 52 & \\
\hline Total & 3 & & \\
\hline
\end{tabular}


7 of the surgery. A total of 25 patients in phenytoin group (92.5\%) had serum phenytoin levels above the minimum therapeutic level of $10 \mu \mathrm{g} / \mathrm{mL}$, with a mean level of $15.83 \pm$ $4.1 \mu \mathrm{g} / \mathrm{mL}$ (- Table 4).

Overall, two patients had drug allergy in the phenytoin group. However, the difference was not statistically significant $(p=0.43)$, as shown in - Table 5. Both patients had maculopapular eruptions involving the torso. None of them had life-threatening adverse drug reactions or signs of toxicity. The serum phenytoin levels in both patients were in the therapeutic range. They, however, improved with no further complications.

At the time of discharge, the majority of the patients had no neurological deficit except four patients who had hemiparesis. At 6-month follow-up, one patient had complete

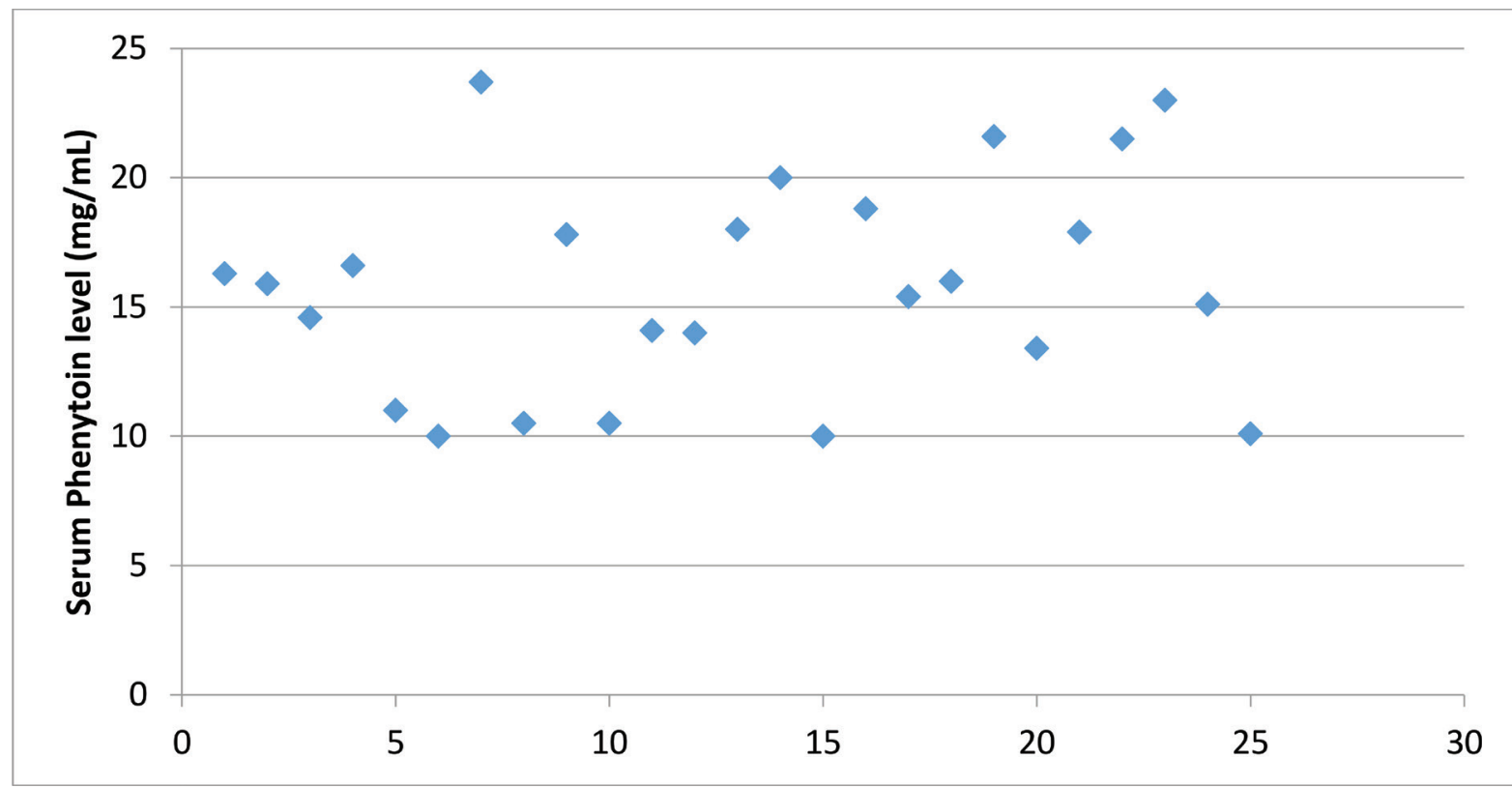

Fig. 4 Serum phenytoin levels determined on postoperative day $7(n=25)$.

Table 4 Comparison of drug reactions in both groups $(n=52)$

\begin{tabular}{|l|l|l|l|}
\hline Groups & Drug reaction & No drug reaction & -Value \\
\hline Phenytoin & 2 & 23 & 0.437 \\
\cline { 1 - 2 } No phenytoin & 0 & 27 & \\
\hline
\end{tabular}

Table 5 Comparison of seizure, mortality, extended Glasgow Outcome Scale (GOSE) and Modified Rankin Scales (MRS) in both groups

\begin{tabular}{|c|c|c|c|}
\hline & \multicolumn{2}{|c|}{ Group } & \multirow[b]{2}{*}{$p$-Value } \\
\hline & Phenytoin & No phenytoin & \\
\hline Seizure & $0(25)$ & $3(27)$ & 0.26 \\
\hline \multicolumn{4}{|l|}{ Mortality } \\
\hline At 1 month & $0(25)$ & $3(27)$ & 0.26 \\
\hline \multicolumn{4}{|c|}{ GOSE at 6 months } \\
\hline GR+ & $15(25)$ & $14(27)$ & 0.55 \\
\hline GR- & $7(25)$ & $10(27)$ & 0.48 \\
\hline MD- & $1(25)$ & $0(27)$ & 0.96 \\
\hline $\mathrm{SD}+$ & $2(25)$ & $0(27)$ & 0.43 \\
\hline $\mathrm{D}$ & $0(25)$ & $3(27)$ & 0.26 \\
\hline \multicolumn{4}{|c|}{ MRS at 6 months } \\
\hline $0-1$ & $22(25)$ & $24(24)$ & \multirow[t]{2}{*}{0.248} \\
\hline $2-3$ & $3(25)$ & $0(24)$ & \\
\hline
\end{tabular}


recovery while three had some residual motor deficit with some improvement.

Overall, mortality in our study was $5.7 \%$. All three cases of death were in the phenytoin group. One patient succumbed after being complicated with subdural empyema. The other two patients had initial GCS of $<8$ and had CT scan features of posterior cerebral artery infarction.

Modified Rankin Scale analysis revealed that the majority of the patients at the time of presentation had moderate to severe disability. Forty-seven (90.3\%) had MRS of $\geq 3$. Only 9.6\% patients were able to look after their own affairs without assistance (MRS $\leq 2)$. All three patients who developed seizure had MRS $\geq 3$ at presentation.

Functional status at 6-month assessment using MRS of the surviving 49 patients revealed that only 2 patients had moderate to severe disability MRS $\geq 3$. The majority of patients $(95.9 \%)$ were able to look after their own affairs without assistance (MRS $\leq 2$ ). None of the patients were in a significant morbid state (MRS $\geq 4)$. No difference was noted in phenytoin or no phenytoin group ( - Table 5) $(-$ Fig. 5).

At 6 months, GOSE analysis revealed $88.4 \%$ of the patients had a score of GR- or GR+, that is, lower good or upper good recovery ( - Fig. 6). No difference was noted in the phenytoin and no phenytoin groups.

\section{Discussion}

Chronic subdural hematoma is a common neurological disease process, especially in the elderly age group with the age-related cerebral atrophy, leading to stretched bridging veins. ${ }^{20}$

More than half of the patients (55.7\%) in our study had some form of comorbidity; and hypertension was found to be the most common among our patients. This is in congruence with the age group most commonly affected by CSDH where the incidence of cardiac-related diseases is higher.
Performance scales such as the MRS and GOSE were used to measure deficits. These scales measure independence rather than performance of specific tasks and in this way incorporate mental as well as physical adaptation to the neurological deficits. The score on such a scale gives a better idea of whether patients can look after themselves in daily life than activities of daily life (ADL) scores, such as Barthel Index, and represent handicap rather than disability. ${ }^{21}$

At admission, MRS was poor in the majority of our cases. Moderate to severe disability was seen in 47 patients (90.3\%). At 6 months following surgery, moderate to severe disability was seen in only 2 of the surviving 49 patients (4.0\%). This showed that surgical management of CSDH patients with burr hole and evacuation even in moderate to severely disabled patients resulted in good outcome. Comparison of MRS at 6 months in the phenytoin and no phenytoin groups showed no statistical difference. At 6 months, GOSE assessment showed a good recovery following surgery in majority of the patients in both groups. The majority of the patients $(88.4 \%)$ were able to return to normal life with or without some symptoms. Three patients (5.7\%) were unable to work and requiring some or no assistance at home. However, there was no statistical difference between the phenytoin and no phenytoin groups in their functional outcome. Similarly, Sabo et al found no difference in the functional outcome with or without the use of phenytoin. ${ }^{12}$

In our study, there were three mortalities in the 30-day period. All of them were in the no phenytoin group. However, the difference was not statistically significant $(p=0.26)$. The cause of death in one patient was subdural empyema. The other two patients presented with poor preoperative status with a GCS of 7; despite prompt evacuation, they failed to improve. Both patients had posterior cerebral artery infarcts prior to surgery. One patient died on day 5 and the other on day 10 . There were no further mortalities in the 6 -month follow-up period.

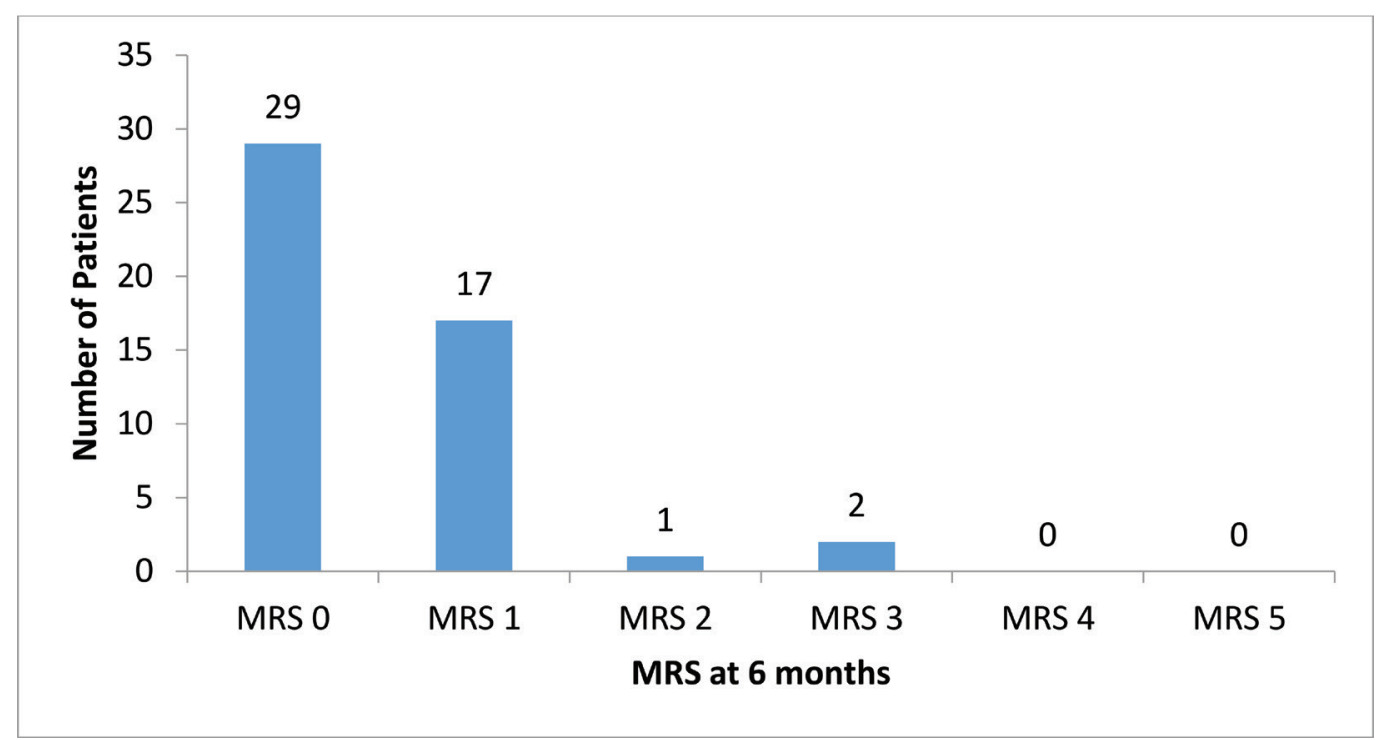

Fig. 5 Functional assessment using Modified Rankin Scale (MRS) at 6 months ( $n=49$ ). 


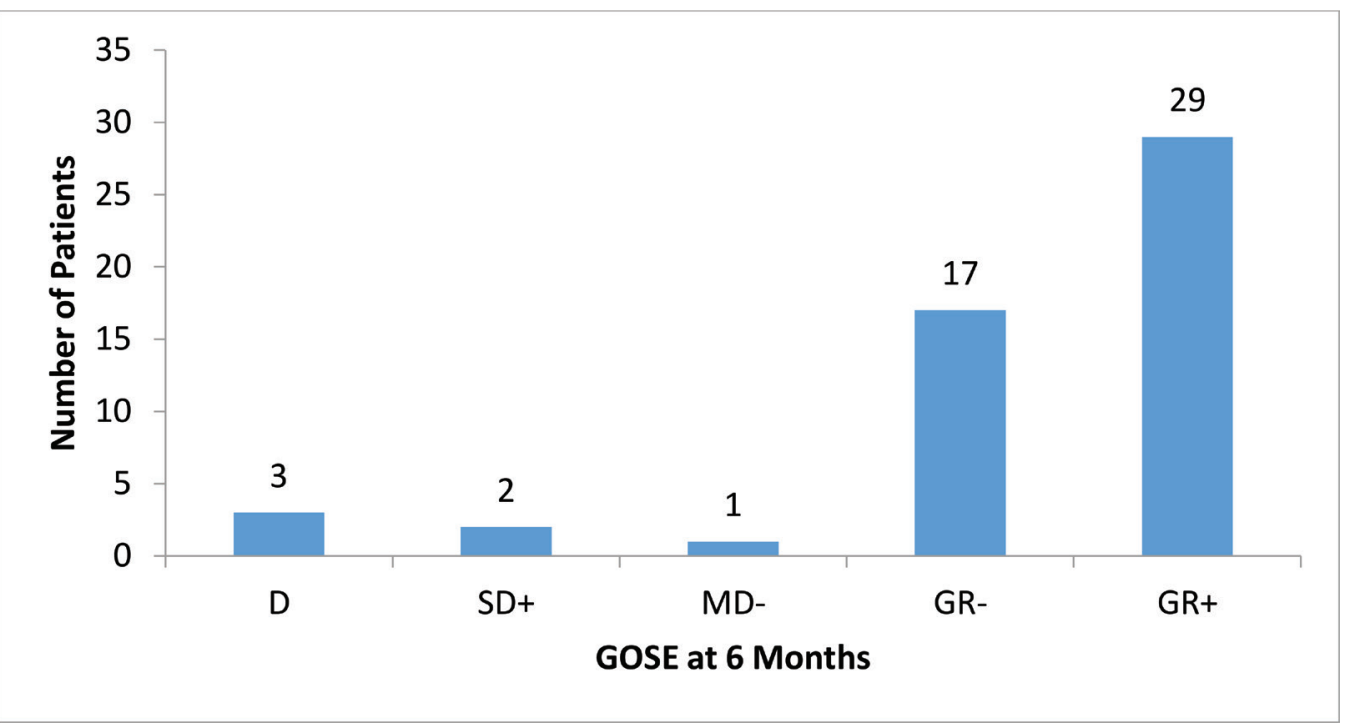

Fig. 6 Functional assessment using Glasgow Outcome Scale (GOSE) at 6 months ( $n=52$ ).

Although several AEDs are available for early posttraumatic seizure (PTS) prophylaxis, phenytoin is used most commonly. ${ }^{22}$ Two class I studies assessed the efficacy of phenytoin for PTS prophylaxis in patients with severe traumatic brain injury (TBI). Temkin et al demonstrated a significantly lower rate of early PTS development among the group who received prophylaxis compared with the placebo group with a relative risk (RR) of $0.25 .^{23}$ Young et al. evaluated a similar phenytoin regimen in a smaller but similar cohort and found no significant difference. ${ }^{24}$ However, the rate of early seizures reported in the study (3.7\%) was much lower than the rates reported in other studies; and the 95\% confidence interval (CI: $0.27-3.58)$ was very wide, suggesting insufficient power to detect a statistical difference. To arrive at a definitive conclusion, Chang et al pooled the data from two class I studies and demonstrated a significantly lower rate of early seizures among the pooled prophylaxis group, compared with the pooled control group with a RR of 0.37 (95\% CI: 0.18$0.74) .^{22}$ Additionally, one class III study evaluated phenytoin and showed a significant difference in PTS risk reduction (RR: 0.24, CI: 0.06-0.98). ${ }^{25}$

In most instances, phenytoin is administered intravenously with a loading dose of $17 \mathrm{mg} / \mathrm{kg}$ intravenous infusion over 30 to 60 minutes, followed by a maintenance dose of $100 \mathrm{mg}$ given three times daily, either intravenously or orally for a total of 7 days. ${ }^{26}$ In our study, $92.5 \%$ of the patients in the phenytoin group had serum phenytoin levels at day 7 within or above therapeutic range. Two patients in the phenytoin group, at day 7 , had serum phenytoin level below the therapeutic drug level despite intravenous loading dose at presentation in the emergency. Dose adjustments were not done as the patients had reached the end of their planned therapeutic period. The two patients with subtherapeutic levels were excluded from analysis. However, none of them had seizures. Temkin et al reported $97 \%$ of phenytoin-treated patients to have levels in or above the therapeutic range on the first day after injury and 57\% maintaining such levels at 1 week. ${ }^{23}$ Young et al observed that more than $78 \%$ of patients maintained therapeutic levels through the first week. ${ }^{24}$

Phenytoin, traditionally, has been linked to serious adverse events, including Stevens-Johnson syndrome, anticonvulsant hypersensitivity syndrome, purple-glove syndrome, and induction of the hepatic cytochrome $\mathrm{P}_{450}$ system, causing significant drug to drug interactions. ${ }^{27,28}$ However, few adverse effects, specifically occurring within the first week of phenytoin therapy, were reported in the studies. ${ }^{23,24}$ Temkin et al reported that $5.2 \%$ of patients stopped phenytoin and $9.2 \%$ stopped placebo in the first week, owing to patient request or idiosyncratic and other reactions. ${ }^{23}$ Young et al reported one patient to experience a rash during the first week of phenytoin therapy. ${ }^{24}$ Debenham et al reported a total of five patients $(0.8 \%)$ who had adverse reactions to phenytoin, including bradycardia, face and trunk redness, skin itchiness without a rash, and elevated liver enzymes. ${ }^{26}$ Sabo et al in their study reported three nonserious dermatological reactions with the use of prophylactic phenytoin. ${ }^{12}$ Rubin noted mild allergic reaction in $7.2 \%$ presenting as a morbilliform rash and $2.4 \%$ acquired phenytoin intoxication..$^{14}$ In our study, two patients in the phenytoin group had adverse effects. However, this was not statistically significant. Both of them developed idiosyncratic reactions to the drug presenting with maculopapular eruptions over the torso. None of them had life-threatening adverse drug reactions. All of them improved after stopping phenytoin. None of the patients on phenytoin showed features of drug toxicity.

Overall incidence of postoperative seizures in our study was $5.7 \%$. The reported incidence of postoperative seizures varies widely from $1.0 \%$ to $23.4 \% .^{10,11}$ The reasons why some studies report such high incidences and the numbers vary so widely, are not clear. A possible explanation could be the use of different operative procedures. Hirakawa et al found that 
postoperative seizures were seen in $5.3 \%$ of patients treated with and irrigation, whereas $23.4 \%$ of patients treated with craniotomy and capsulectomy had seizures. ${ }^{11}$ Our incidence of low postoperative seizures correlates with the overall low incidence found by Rubin (4.5\%) and Ohno (1.5\%). ${ }^{13,14}$ In both series, majority of the patients were treated by burr hole craniostomy. However, it contrasts with the study by Sabo et al, where the incidence of postoperative seizure, although treated with a burr hole craniostomy in the majority, was quite high $(18.5 \%) .^{12}$

Out of the 138 patients studied retrospectively by Rubin et al in 1993, seizures were noted in $4.8 \%$ of patients treated with prophylactic phenytoin compared with $3.4 \%$ in patients without the drug..$^{14}$ In the retrospective analysis by Ohno et al, in 1993, 129 patients treated for CSDH were studied. None of the 73 patients who were given prophylactic AED treatment developed seizures. Only two of 56 (3.5\%) patients who were not given prophylaxis developed early postoperative seizures. ${ }^{13}$ In contrast, Sabo et al, in 1995, reported $2.4 \%$ of incidence of seizures in patients treated with phenytoin compared with $32 \%$ who did not receive prophylactic phenytoin in a retrospective study including 92 patients. ${ }^{12}$ Other retrospective studies have also considered the incidence of seizures in patients with CSDH but have not included any control group for anticonvulsant therapy. ${ }^{3,11}$ In our study, it is notable that all cases of seizures occurred in the no phenytoin group. However, there was no statistical difference showing a beneficial role of phenytoin in preventing postoperative seizures after burr hole drainage for CSDH.

Among the three patients who developed postoperative seizures, one had a postoperative empyema, another patient had a small parenchymal contusion beneath the burr hole site, which was likely iatrogenic, and the last one had no additional findings in the postoperative computed tomography (CT) scan. Although it has been stated that the hematoma or the capsule of the hematoma may play an important role in the incidence of epilepsy, ${ }^{29}$ we consider that seizures in patients with chronic subdural hematoma are rarely related to the encapsulated hematoma itself, but rather to accompanying cerebral insults due to the surgical technique or a complication. ${ }^{30}$

All seizures in our study group occurred within the first 7 days after surgery. None of the surviving patients had further episodes of seizure in the following months. None of them had residual neurological deficits. Grobelny et al noted no difference in clinical outcome of patients having postoperative seizures as compared with those who did not have postoperative seizures. ${ }^{19}$ In contrast, Sabo et al found a significant increase in morbidity and mortality associated with respiratory complications and status epilepticus in patients with the onset of new seizures postoperatively. ${ }^{12}$

Regarding the use of prophylactic anti epileptics in neurosurgical patients, there are various studies for other brain conditions such as tumors and subarachnoid hemorrhage (SAH). In a Cochrane review done in 2008, the authors concluded that the evidence is neutral, neither for nor against seizure prophylaxis, in people with brain tumors. ${ }^{31}$ According to another systematic review of major RCTs, the administration of prophylactic AEDs after brain tumor resection shows no significant reduction in the incidence of seizures compared with that in controls. ${ }^{32}$

Similarly, regarding prophylactic antiepileptics in SAH where it is commonly used, propensity score-matched analysis suggests that prophylactic AEDs do not significantly reduce the risk of seizure occurrence. ${ }^{33}$

Owing to these recent articles doubting the usefulness of prophylactic antiepileptics in other brain conditions as well, we had undertaken the study on a small scale. However, there are various subgroups in chronic SDH such as small/ large, acute component present or absent, alcoholic versus nonalcoholic patients, young/old. These need to be taken into consideration by a larger study with subgroup analysis to find out who actually are the group of patients that would benefit from prophylactic antiepileptics.

\section{Limitations}

Subclinical seizures in ventilated patients in the postoperative period could have been missed, as continuous postoperative electroencephalography was not done in this patient population. Daily serum phenytoin levels of the patients in the phenytoin group and dose adjustment to keep it within the therapeutic range was not done. This could not be done for various practical limitations. This would have given evidence that the patients in the phenytoin group actually had remained in the therapeutic range throughout the 7-day postoperative period.

In our single center study, the incidence of postoperative seizure after burr hole drainage of CSDH was considerably low and the number of patients was relatively small. Sample size was estimated using the incidence of postoperative seizure in the phenytoin and no phenytoin groups in the study by Sabo et al. ${ }^{12}$ Using the incidence of seizures in studies by Ohno et $\mathrm{al}^{13}$ or Rubin et al, the calculated sample size would have been very large. Enrollment of such a large number of patients in the limited study period of 1 year in the study center was not feasible. Therefore, further large-scale, multicentric population studies will be required to address this issue in a more conclusive manner.

\section{Conclusions}

Incidence of postoperative seizures in patients undergoing burr hole drainage for CSDH was low. Routine prophylactic use of AED, namely phenytoin, did not reduce the seizure occurrence in surgically managed patients with CSDH. Furthermore, even though not statistically significant, there was an increased incidence of adverse drug reactions in patients receiving phenytoin. Using GOSE and MRS, the functional outcome of patients, whether receiving phenytoin or not, did not show any statistical difference. Six months' outcome of patients who had postoperative seizure was not different from those who did not have seizure. 


\section{Recommendation}

We cannot make a strong recommendation for or against use of prophylactic antiepileptic in CSDH as the sample size of the study is small. However, the study does not support the routine use of prophylactic phenytoin in prevention of early postoperative seizures in patients treated with burr hole for $\mathrm{CSDH}$, unless the postoperative scan shows a convincing reason to start it.

\section{Conflict of Interest}

None declared.

\section{References}

1 Chen JC, Levy ML. Causes, epidemiology, and risk factors of chronic subdural hematoma. Neurosurg Clin N Am 2000;11(3):399-406

2 Adhiyaman V, Asghar M, Ganeshram KN, Bhowmick BK. Chronic subdural haematoma in the elderly. Postgrad Med J 2002;78(916):71-75

3 McKissock W, Richardson A, Bloom W. Subdural hematoma. Lancet 1960;275(7139):1365-1369

4 Asghar M, Adhiyaman V, Greenway MW, Bhowmick BK, Bates A. Chronic subdural haematoma in the elderly-a North Wales experience. J R Soc Med 2002;95(6):290-292

5 Bourgeois P, Sleiman M, Louis E, et al. L'hématome sous-dural chronique chez les patients de plus de 80 ans [Chronic subdural hematoma in patients over 80 years of age]. Neurochirurgie 1999;45(2):124-128

6 Cousseau DHEMG, Echevarría Martín G, Gaspari M, Gonorazky SE. Hematoma subdural crónico y subagudo. Estudio epidemiológico en una población cautiva [Chronic and subacute subdural haematoma. An epidemiological study in a captive population]. Rev Neurol 2001;32(9):821-824

7 Victor M, Ropper A, Raymond D, eds. Adams and Victor's Principles of Neurology New York: McGraw-Hill; 2001 463-464

8 Santarius T, Hutchinson PJ. Chronic subdural haematoma: time to rationalize treatment? Br J Neurosurg 2004;18(4):328-332

9 Weigel R, Schmiedek P, Krauss JK. Outcome of contemporary surgery for chronic subdural haematoma: evidence based review. J Neurol Neurosurg Psychiatry 2003;74(7):937-943

10 Grisoli F, Graziani N, Peragut JC, et al. Perioperative lumbar injection of Ringer's lactate solution in chronic subdural hematomas: a series of 100 cases. Neurosurgery 1988;23(5):616-621

11 Hirakawa K, Hashizume K, Fuchinoue T, Takahashi H, Nomura K. Statistical analysis of chronic subdural hematoma in 309 adult cases. Neurol Med Chir (Tokyo) 1972;12(0):71-83

12 Sabo RA, Hanigan WC, Aldag JC. Chronic subdural hematomas and seizures: the role of prophylactic anticonvulsive medication. Surg Neurol 1995;43(6):579-582

13 Ohno K, Maehara T, Ichimura K, Suzuki R, Hirakawa K, Monma S. Low incidence of seizures in patients with chronic subdural haematoma. J Neurol Neurosurg Psychiatry 1993;56(11):1231-1233

14 Rubin G, Rappaport ZH. Epilepsy in chronic subdural haematoma. Acta Neurochir (Wien) 1993;123(1-2):39-42
15 Ratilal BO, Pappamikail L, Costa J, Sampaio C. Anticonvulsants for preventing seizures in patients with chronic subdural haematoma. Cochrane Database Syst Rev 2013; (6):CD004893

16 Ohaegbulam SC. Surgically treated traumatic subacute and chronic subdural haematomas: a review of 132 cases. Injury 1981;13(1):23-26

17 Temkin NR. Risk factors for posttraumatic seizures in adults. Epilepsia 2003;44(s10):18-20

18 Ferreri S, Roth MT, Casteel C, Demby KB, Blalock SJ. Methodology of an ongoing, randomized controlled trial to prevent falls through enhanced pharmaceutical care. Am J Geriatr Pharmacother 2008;6(2):61-81

19 Grobelny BT, Ducruet AF, Zacharia BE, et al. Preoperative antiepileptic drug administration and the incidence of postoperative seizures following bur hole-treated chronic subdural hematoma. J Neurosurg 2009;111(6):1257-1262

20 Hubbard BM, Anderson JM. A quantitative study of cerebral atrophy in old age and senile dementia. J Neurol Sci 1981;50(1):135-145

21 van Swieten JC, Koudstaal PJ, Visser MC, Schouten HJ, van Gijn $\mathrm{J}$. Interobserver agreement for the assessment of handicap in stroke patients. Stroke 1988;19(5):604-607

22 Chang BS, Lowenstein DH; Quality Standards Subcommittee of the American Academy of Neurology. Practice parameter: antiepileptic drug prophylaxis in severe traumatic brain injury: report of the Quality Standards Subcommittee of the American Academy of Neurology. Neurology 2003;60(1):10-16

23 Temkin NR, Dikmen SS, Wilensky AJ, Keihm J, Chabal S, Winn HR. A randomized, double-blind study of phenytoin for the prevention of post-traumatic seizures. N Engl J Med 1990;323(8):497-502

24 Young B, Rapp RP, Norton JA, Haack D, Tibbs PA, Bean JR. Failure of prophylactically administered phenytoin to prevent early posttraumatic seizures. J Neurosurg 1983;58(2):231-235

25 Heikkinen ER, Rönty HS, Tolonen U, Pyhtinen J. Development of posttraumatic epilepsy. Stereotact Funct Neurosurg 1990;5455:25-33

26 Debenham S, Sabit B, Saluja RS, et al. A critical look at phenytoin use for early post-traumatic seizure prophylaxis. Can J Neurol Sci 2011;38(6):896-901

27 Jones GL, Wimbish GH, McIntosh WE. Phenytoin: basic and clinical pharmacology. Med Res Rev 1983;3(4):383-434

28 Sajanti J, Majamaa K. High concentrations of procollagen propeptides in chronic subdural haematoma and effusion. J Neurol Neurosurg Psychiatry 2003;74(4):522-524

29 Kotwica Z, Brzeiński J. Epilepsy in chronic subdural haematoma. Acta Neurochir (Wien) 1991;113(3-4):118-120

30 Tindall GT, Payne NS II. O'Brien MS. Complications of surgery for subdural hematoma. Clin Neurosurg 1976;23:465-482

31 Tremont-Lukats IW, Ratilal BO, Armstrong T, Gilbert MR. Antiepileptic drugs for preventing seizures in people with brain tumors. Cochrane Database Syst Rev 2008;(2):CD004424

32 Chandra V, Rock AK, Opalak C, et al. A systematic review of perioperative seizure prophylaxis during brain tumor resection: the case for a multicenter randomized clinical trial. Neurosurg Focus 2017;43(5):E18

33 Panczykowski D, Pease M, Zhao Y, et al. Prophylactic antiepileptics and seizure incidence following subarachnoid hemorrhage: a propensity score-matched analysis. Stroke 2016;47(7):1754-1760 